\title{
Optimising Qualitative Longitudinal Analysis: Insights from a Study of Traumatic Brain Injury Recovery and Adaptation
}

Joanna K Fadyl ${ }^{1}$, Alexis Channon ${ }^{1}$, Alice Theadom ${ }^{1,2}$, and Kathryn M McPherson ${ }^{1}$ on behalf of the TBI Experiences Research Group.

${ }^{1}$ Centre for Person Centred Research, Auckland University of Technology

${ }^{2}$ National Institute for Stroke and Applied Neurosciences, Auckland University of Technology

Corresponding author:

Joanna Fadyl, Auckland University of Technology, Private Bag 92006, Auckland 1142

Email: jfadyl@aut.ac.nz; +64 99219999 x 7675

TBI Experiences Research Group:

Kathryn McPherson; Alice Theadom; Joanna Fadyl; Laura Wilkinson-Meyers; William Levack; Nicola Starkey; Nicola Kayes; Valery Feigin; Matire Harwood; Grant Christie.

Publisher's version available at: Fadyl, J. K., Channon, A., Theadom, A., \& McPherson, K. M. (2016). Optimising Qualitative Longitudinal Analysis: Insights from a Study of Traumatic Brain Injury Recovery and Adaptation. Nursing Inquiry. doi:10.1111/nin.12170 
Acknowledgements:

Funding for the research was provided by the Health Research Council of New Zealand. We were also awarded a grant from the Faculty of Environmental Sciences, Auckland University of Technology as financial support for preparation of the article.

We acknowledge the following people who have provided valuable contributions to the project. We thank the participants for their time and energy in sharing their experiences. Input into the design or running of the project was contributed by William Levack, Nicola Kayes, Laura Wilkinson-Meyers, Nicola Starkey, Grant Christie, Valery Feigin, Kay Shannon, Kirsten Thomas, Dawn Willix-Payne, Claire Townson, Elisa Lavelle, Margaret Dudley, and Thivasha Naidoo-Melis, the organisations that facilitated participant recruitment, and the community reference group: Stephen Jenkins, Matire Harwood, Alison Forster and Suzanne Mudge. Greta Smith contributed financial management, data modelling and administrative support. Rean Fadyl and AcumenBI provided consultancy regarding database design and data visualisation software and development.

Declaration of conflicting interests:

The authors of this article came to be aware of the particular software chosen by the research team for data visualisation through a family relationship with a consultant who sells and configures the software for local businesses. They are one of many consultancies worldwide that deals in this software, and the desktop licence for the software is available from the software developer at no charge in the current pricing model. 


\section{Abstract}

Knowledge about aspects that influence recovery and adaptation in the post-acute phase of disabling health events are key to understanding how best to provide appropriate rehabilitation and health services. Qualitative longitudinal research makes it possible to look for patterns, key time points and critical moments that could be vital for interventions and supports. However, strategies that support robust data management and analysis for longitudinal qualitative research in healthcare are not well documented in the literature. This article reviews three challenges encountered in a large longitudinal qualitative descriptive study about experiences of recovery and adaptation after traumatic brain injury in New Zealand, and the strategies and technologies used to address them. These were: 1) tracking coding and analysis decisions during an extended analysis period; 2) navigating interpretations over time and in response to new data; and 3) exploiting data volume and complexity. Concept mapping during coding review, a considered combination of information technologies, employing both cross-sectional and narrative analysis, and an expectation that sub-analyses would be required for key topics helped us manage the study in a way that facilitated useful and novel insights. These strategies could be applied in other qualitative longitudinal studies in healthcare inquiry to optimise data analysis and stimulate important insights.

\section{Keywords}

Qualitative methods; Qualitative Longitudinal; Data Analysis; Data Management 


\section{Introduction}

Specific knowledge about aspects that influence a person's recovery and adaptation after disabling health events are key to understanding how best to provide appropriate rehabilitation and healthcare (e.g. see Becker, 1993; Lowe \& Molassiotis, 2011). Qualitative studies have the ability to provide insight into the ways in which people experience and construct their recovery and adaptation, and thus inform the development of novel interventions designed to facilitate positive trajectories (Campbell et al., 2007). Studies that are targeted to specific issues or homogeneous populations can draw important insights from short-timeframe, cross-sectional studies. However, if the goal is the development of rehabilitation interventions that can influence longer-term recovery and adaptation in a heterogeneous population, a larger participant sample and longitudinal analysis is needed (Charmaz, 1995; Levack, Kayes, \& Fadyl, 2010). Qualitative longitudinal research in particular has been argued to improve the potential for capturing the complexity of social phenomena by accounting for movements with regard to experiences over time (Bidart, 2013). When considering recovery and adaptation, this makes it possible to look for patterns, key time points and critical moments that could be useful when reviewing and designing rehabilitation interventions and supports.

Longitudinal qualitative designs have been used to investigate a range of different human experiences and processes. Longitudinal qualitative work is common in the social sciences, and there are an increasing number of studies that use this method to illuminate illness or recovery trajectories for the purposes of informing healthcare or rehabilitation (for recent examples see Faircloth, Rittman, Boylstein, Young, \& Van Puymbroeck, 2004; Lowe \& Molassiotis, 2011; Pinnock et al., 2011). Most of the methodological discussion regarding strategies for optimising analysis in longitudinal qualitative studies still relates to sociologically-focused studies. Although this information is relevant, there are key 
features of clinically-focused studies that are missing in these discussions. For example the ups and downs associated with the health event itself, and the journey of recovery and adaptation, along with the severe disruption to 'normal' life entailed in this process (Becker, 1993; Faircloth, Boylstein, Rittman, Young, \& Gubrium, 2004; Levack et al., 2010). Furthermore, there are characteristics of the study populations in clinically-focused studies that are specific to the context - for example in the study described below, our participants were dealing with very recent and significant changes to their functioning and life routines. As such, they were in a period of very frequent re-evaluation of their life experiences and interpretations.

As discussed by Thompson and Holland (2003), qualitative longitudinal studies are highly labourintensive. The time-consuming nature of qualitative research is made more complicated with the element of temporality, especially when seeking to capture within-person changes and patterns within the sample population in the analysis process (Calman, Brunton, \& Molassiotis, 2013; Thomson \& Holland, 2003). The study we undertook (TBI Experiences Study) was a qualitative descriptive study to capture a range of experiences of recovery and adaptation from a diverse traumatic brain injury (TBI) population, including the change in these experiences over time. It aimed to follow 50 people who had a TBI and their significant other(s) over three interviews from 6 months to 24 months post-injury. In designing and executing the study, the research team frequently sought information from the literature to help manage various issues. These issues concerned the longitudinal design of the study; the volume of data; and the complexities of analysis when seeking to capture important aspects both in specific phases of recovery and in change over time. We were able to draw on existing information in the literature to inform the study design in the areas of planning, participant engagement and interviewing techniques (e.g. Carlsson, Paterson, Scott-Findlay, Ehnfors, \& Ehrenberg, 2007; Charmaz, 2006; Corden \& Millar, 2007; Lloyd, Gatherer, \& Kalsy, 2006; Rubin \& Rubin, 2005; Thomson, 2007; Thomson \& 
Holland, 2003). However, aspects relating to data management and the complexity of analysis were much more challenging to operationalise. The purpose of this paper is to outline key challenges that we encountered during the research, and the considerations and methods employed in addressing these to optimise data analysis. The findings we present aim to extend the limited exploration of these issues in the literature - not just in relation to $\mathrm{TBI}$, but to clinically-focused longitudinal qualitative inquiry generally.

\section{Design of the qualitative descriptive longitudinal study}

\section{Overview}

The study we undertook sought to explore recovery and adaptation from disabling TBI from the perspective of injured person and significant other(s) (partner, family, friend), over the first two years post injury. Specific research questions explored: 1) the outcomes of importance over time; and 2) the key barriers and facilitators to recovery and adaptation; as perceived by the participants.

Our longitudinal qualitative descriptive study was underpinned by a post-positivist epistemology and social constructionist perspective (Patton, 2002), utilizing data collection and analysis methods described by Charmaz (2006). The injured participants were people over 16 years who had experienced a TBI classed as severe, moderate, or mild, and had persistent problems (emotional, cognitive or physical difficulties) at 6 months post-injury. Significant others (people closely connected with the injured individual and their recovery) were also invited to take part in either a dyadic interview (with the person with a TBI) or a separate interview according to preference of both parties. 


\section{Study team structure}

The study team was comprised of health professionals and academics in various health disciplines, and contained several layers with different responsibilities. A core team was involved in the day-to-day execution of the study, including participant contact, scheduling interviews, data management, coding, analysis, and documentation of processes and decisions. The interviews were conducted by members of the core team and research assistants trained in a health profession and in qualitative interviewing. In addition to this, a steering group of senior researchers met every 3-4 months to review key developments and discuss decision-making. A sub-group of the steering group also met at six-monthly intervals during the study to discuss the analysis specifically. The authors were all involved at each of these layers. Finally, a community reference group made up of people with personal experience of TBI and health professionals who work people experiencing TBI met with the core study team at several points throughout the study in order to provide input into decisions and provide consultation on application and dissemination of study findings.

\section{Data collection}

Data collection was through serial semi-structured interviews at approximately 6, 12 and 24 months post injury (variation of up to 3 months following specified time-points was expected). Interviews were conducted with 52 people with TBI and 37 significant others. A total of 152 interviews were conducted over the three time-points. Forty-eight of these interviews were with the injured person and their significant others together. Seventy-nine interviews were with the injured person only, and 25 were with the significant other only. Not all participants were able to be interviewed at all timepoints: 31 TBI participants (60\%) completed all 3 interviews. Serial interviews enabled previously discussed issues to be explored at subsequent time-points - to review changes, new perspectives and insights, as well as 
exploring new issues and events that had occurred in the intervening period. The interview guide allowed for prior interviews and preliminary analysis to inform the planned avenues of inquiry. The study database (see description later in the article) allowed the team to separately identify comments made by TBI or 'significant other' participants even within the same interview, in order to ascertain which issues were primarily raised by the person with TBI and which issues were primarily identified by close family and/or friends.

\section{Data analysis}

Data coding and analyses were undertaken concurrently with recruitment and interviewing in order to identify emerging themes and enable subsequent interviews to explore areas that needed more information for development. In particular, patterns identified during early analysis often required further inquiry to clarify how they manifested (or not) in different situations and with different types of people.

The 6-month interview data was initially analysed separately to capture the experience of the acute stage of injury and also inform the ongoing interviews. Initial coding of the transcribed interviews involved generating phrases that captured the experiences described by participants relating to processes of and influences on recovery and adaptation (e.g. "having a comparison point can be helpful"). Once initial coding was completed for the 6 month interviews, reports capturing the interview extracts that were allocated to each code and key participant and context information were generated. Three of the core researchers ([initials - removed for peer review]) formed an analysis working group, who met for a series of focused review sessions to interrogate these initial codes, using constant comparison (Charmaz, 2006) to review and refine codes based on more interpretive ideas and themes that were emerging from the analysis. 
Following this process for the 6-month data, transcripts from all three interview time-points (or subset if data from one interview timeframe were missing) were grouped for each participant and read in chronological order. The 12-month and 24-month transcripts were then coded with reference to the 6month codes to capture aspects that had changed or remained the same from the previous interview(s) to reflect temporality of experiences within each participant. Codes generated from 12-month and 24month interviews were then compared across participants to encompass differences and similarities across the sample. Code names and descriptions were reviewed again at this stage to reflect the longitudinal data. New codes emerging at the later time-points but not reflected in the 6-month codes were also added. All codes were then incorporated into the larger thematic analysis. For example, one of the new codes created at this stage was "revisiting, reviewing and revising", which incorporated discussion of the ways in which notions of what constituted progress (and also appropriate services) were continually reflected on and revised as part of the recovery process.

\section{Key challenges in optimising the qualitative longitudinal data}

\section{analysis}

As expected in a population of people experiencing a long-term recovery trajectory, participants in this study experienced many ups and downs in their recovery and adaptation during the course of the study. Some participants experienced additional injuries, other health issues, or major life events that affected them and their recovery significantly. The processes of data analysis therefore needed to acknowledge the unpredictable course that recovery and adaptation may take within the context of other aspects of life, while retaining the ability to explore specific issues that occurred at key moments and more general 
patterns over time. Key challenges that we encountered in the design and execution of the study because of these factors are discussed below. We also show how we addressed each challenge.

\section{Tracking coding and analysis decisions during an extended analysis period}

Our study design required that data analysis decisions be made over an extended period of time (i.e. years). During this time multiple decisions concerning code creation, naming and refinement, and allocations of interview extracts to codes and of codes to thematic groupings were required. It was crucial that such decisions and associated discussion were recorded to facilitate review, reflexivity and auditability. Furthermore we were aware that staff responsibilities within the study might (and in fact did) shift, and interviews at later time-points triggered different views and interpretations of earlier interviews (Corden \& Millar, 2007; Thomson \& Holland, 2003). Finally, because the study was clinicallyfocused in its aims, it was crucial that the analysis and interpretation was focused on responding to the needs of stakeholders (people affected by TBI and their health professionals), as reflected in the research question.

During discussion about the most appropriate data management processes and systems, we identified four important features that were required. These are listed below.

- Ability to track and review all analysis decisions and associate these decisions with codes and interview extracts - including being able to review disused codes and see when and where interview extracts had been re-allocated due to analysis decisions.

- Ability to visualise layers of analysis - looking at both timepoint-specific issues and changes over the course of time since the injury that were pertinent and relevant to stakeholders. 
- $\quad$ Ability to store large volumes of interview extracts and easily associate them with many characteristics of the interviewee and interview context, which interview in the series it was, time since the injury, and coding decisions from analysis.

- $\quad$ Flexibility to draw out this information in various different combinations, in formats that could easily reviewed manually or imported into software that would facilitate exploration of the data (e.g. data visualisation software).

We developed processes for coding and review alongside systems for data storage and access that would support those processes and meet the identified needs.

\section{Coding and review processes}

Following preliminary coding of interviews, we agreed on a set of procedures for the creation and review of more interpretive codes. This involved group discussion in which firstly a series of preliminary codes were identified as having crossovers or similarities. Following this, each interview extract allocated to that series of codes was discussed, followed by review of the allocation, names and descriptions of the individual codes (including 'retiring' codes where appropriate). The process of reviewing and retiring preliminary codes was often reflected in the description of the interpretive code. For example, after comparison and discussion of all the extracts in a list of codes that appeared to relate to monitoring as a form of support, the description of this code gives a detailed summary of its contents and notes that it contains extracts that were "re-coded" from the preliminary (now retired) codes:

"noticing symptoms" and "trying to help while trying to protect", and retains the original descriptions of these codes for future reference.

Principles for naming and describing codes were adopted to ensure they captured the substance of the range of interview extracts allocated to that code, and addressed the research focus regarding what 
helps and hinders adaptation and recovery. Full descriptions of each code were recorded in addition to the short code name to ensure there was always sufficient detail regarding what the code represented. Guided by Charmaz (2006), we chose to focus on code names that represented either conditions participants found themselves subject to (e.g. "life on hold") or actions they chose to take in response to their situation (e.g. "picturing a future"). The coding aimed to capture the range of experiences described by the diverse sample, thus not every code was applicable to every participant's situation. Rather, they described the scope of conditions and actions that were characteristic of TBI recovery and adaptation in our sample and aimed to capture the range of experiences (positive and adaptive or negative).

Progress, decisions and quandaries were documented both in the form of meeting records and note fields in the study coding database linked to the coding decision or code description entries they were associated with. This documentation was regularly discussed with the most senior principal investigator [initials removed for peer review], and also presented to the analysis group from the study steering group at regular intervals to test the emerging ideas. To assist in ensuring the data interpretations were presented in a way that would facilitate translation to practice, a community reference group was established including service providers and people who had experienced a TBI. Key ideas from the analysis were presented to the community reference group at the end of each stage, in order to generate feedback regarding interpretation, appropriate follow-up and routes for dissemination

\section{Mapping code grouping (concept mapping)}

The analysis team also used a manual mapping technique to track changes in code grouping and associations between codes and groups during the later stages of analysis. Codes were grouped to represent overarching notions that connected them (e.g. "allowing me to change what I normally do to 
manage symptoms and recover"), but these groupings changed and developed as the analysis progressed. The mapping technique involved creation of individual items representing each code, which could then be moved around on a board, and associations drawn between them to consider different thematic groupings. This process allowed the analysis team to visualise how the links and relationships between codes and groups of codes led to the identification of overarching themes, and how these links and relationships changed as new data were added and interpretations were adjusted in response (see also section on "navigating interpretations" below). Photographs were taken of the boards at the end of each analysis session, creating a record of the way the code names, grouping and associations changed over the extended analysis period. Digital maps were created from the photographs to represent key phases of analysis.

\section{Data management system}

Although qualitative data management software packages were available, none fulfilled all the criteria we identified as important for data management in our study (articulated in the four required features listed above). In brief, we could not identify a package that would allow us to link together all the related information about the interviews, the participants and their relationships, the coding and analysis decisions and enable reporting of every possible combination to facilitate review for constant comparison and analysis without the need to export and rearrange data, which would be timeconsuming and open up scope for potential errors. We also needed a system that would enable us to do a large amount of reviewing and recoding while still retaining all the information about 'retired' codes and the extracts they contained, as required by our analysis review procedures described above. Thus we used a combination of a bespoke database and data visualisation software. Unlike generic qualitative data management and analysis packages, which are useful in a range of other contexts, the design of the 
bespoke database was able to be very simple because that it was created to address only the needs of this specific study. It was designed to provide easy and flexible reporting for analytic review - allowing any combination of associated factors to be drawn together in a report (for example: code allocation of extracts organised by interview time-point, participant ID and injury mechanism; code allocation of extracts for a sub-group of codes with participant characteristics information). Furthermore, results could be exported to a simple format for importing into data visualisation software. The database also allowed for extensive notes regarding decisions about individual extracts and entire codes, and required that these notes were made as part of the process of code creation or retirement (allowing us to revisit these decisions if necessary). The database was first produced using Apache OpenOffice Base software by two members of the study team [initials removed for peer review] and a consultant business analyst, and later recreated on a Microsoft SharePoint 2013 platform by university information technology staff to enable greater stability, better user access and faster re-coding of extracts from retired codes.

\section{Navigating interpretations over time in response to new data}

As noted by other researchers who have discussed processes of analysis in longitudinal qualitative inquiry (Corden \& Millar, 2007; Thomson \& Holland, 2003), a crucial challenge was navigating interpretations of the data as analysis became mediated by additional data from the same participants, in their re-telling and re-evaluating experiences over the passage of time. A further challenge in this study was related to the fact that the topic of inquiry was recovery and adaptation, which inherently involves re-interpretation and re-evaluation of experiences. We wanted to capture this process of change over time without losing insights about what helped and hindered recovery and adaptation for people at particular points and stages following their injury. 
In developing an analysis approach that allowed for both of these types of insights, we took the position that two types of knowledge would be equally valued, even when one appeared to contradict the other at a later time. These were a) retrospective knowledge based on participant evaluation of experiences and personhood, and b) in-the-moment knowledge based on current needs and experiences (referred to as retrospective hindsight and contemporaneous insight by Thompson and Holland (2003). Thompson and Holland (2003) discuss how in their study, interpretation was always provisional because the next round of data collection could challenge it. While aspects of our interpretation were provisional in the sense that later aspects could shift the overall picture, there was a need to close off certain parts of analysis to ensure insights important at a given time retained their position in the overarching analysis. For example, while conducting the analysis, we found that early descriptions from participants of the situations they found themselves in, and the actions they took in response to these situations, were of considerable importance in how they experienced their recovery. This was the case even when these experiences were later reinterpreted or reappraised by the participant. Thus, we decided it was crucial to retain a version of the analysis that focused entirely on the 6-month data, which could be reported separately in order to highlight experiences and effective strategies and supports [reference forthcoming, removed for peer review]. This 6-month analysis also provided a baseline point for an examination of change over time, when combined with the data from later time-points.

Thompson and Holland (2003) and White and colleagues (2012) proposed that "narrative" / "vertical" (within participant) and "cross-sectional" / "horizontal" (across participant) analyses are both needed to get a coherent and nuanced understanding of the data in a longitudinal qualitative inquiry. We found this to be a useful strategy for retaining time-point-specific insights, while also enabling analysis of change over time. We slightly adapted the process described by Thompson (2007) to create narrative case histories, as well as conducting cross-sectional analysis of all participant interviews. Thompson 
(2007) discussed the value of creating a limited number of detailed case histories to contrast with each other. Our approach was to work with 'case sets' for each participant in the later stages of analysis - a case set being up to 6 interviews that were associated with a particular person with a TBI (including all time-points and sometimes also separate significant other interviews). During this stage of analysis, a case set was created, developing a narrative summary of the experiences and insights from that person's series of interviews. Following this, the case set was compared with the overall cross-sectional analysis from the 6-month data set to identify aspects that provided further detail or nuance in relation to how things shifted over time. This approach felt more appropriate than broad cross-sectional analysis for examining individual movement in relation to situations and responses to them. While the crosssectional analyses were important to gather broad insights about recovery and adaptation at different times since the injury, it was more difficult to see the processes of change with a cross-sectional view.

\section{Exploiting data volume and complexity}

Drawing on insights from existing literature concerning large qualitative studies, we were mindful that we would be dealing with a large volume of data, and that data was from a relatively heterogeneous population collected over years, intensifying its complexity. We felt there was a risk of inadvertently simplifying the data and interpretations in order to be able to work with such a large volume of multifaceted information. Thus, we were keen to ensure the volume and complexity of data would be utilised by building in analysis strategies designed to help see the complexity of the data and also allow us to follow up key insights while still producing overall study findings that were able to be reported in a coherent way. These strategies were concept mapping during coding review, the use of data visualisation software, and pursuing separate sub-analyses of particular topics. They are described in detail below. 


\section{Concept mapping with coding review}

As mentioned previously, we used a manual mapping technique to track code grouping and associations between codes and groups. This allowed us to work with 'provisional' groupings and discuss the emergence of higher-level concepts during the coding review sessions. It also enabled us to refer to a visual representation of the analysis of the entire dataset while reviewing individual codes and the extracts that were allocated to those codes. This was extremely helpful for keeping in mind how the detail we were reviewing at the time related to the whole, and in particular how specific extracts or interviews extended or challenged our current 'working' interpretations.

\section{Data visualisation}

Following analysis of the interviews up to 24 months, all information contained in the database (code names and descriptions, coded interview extracts, interview factors, participant demographic and contextual information) was imported into interactive information visualisation software QlikSense (Qlik, 2015). This enabled us to easily see patterns in relative prominence of codes over the three timepoints both overall and within categories and link directly back to the relevant interview extracts (sections of transcripts) to see what those patterns illuminated. It also allowed us to identify potential patterns between thematic content of the interviews and demographic characteristics of participants that could be investigated further within the data. Below we give two examples of how data visualisation augmented our analysis. To provide an example of how the data visualisation was set up, we also include a snapshot of the "code explorer sheet" showing the relative prominence graphic (on the left hand side of the screen) as a supplement to this article. The time points are in chronological order from earlier to later. Darker colour represents greater prominence of the code compared with the 
other codes and time points. The right-hand side of the screen contains another graphic that was set up for exploring demographics in the coded data.

\section{Illuminating nuances in change in experiences over time}

Visualising the relative prominence of codes across all participants at the different time-points showed that some codes remained consistently prominent throughout all three time-points, while other codes were more associated with earlier or later post-injury. While it would be inappropriate to interpret these patterns at face value, easily accessible links back to the interview extracts for review enabled us to see what, if anything, these patterns illuminated in the data. For example, within the category "allowing for the course of recovery" we could see a pattern showing that the prominence of particular codes in the coding of extracts from early interviews differed from later interviews. In reviewing the associated interview extracts, we could see that a focus on letting go of expectations of control and allowing time to enable recovery to occur was dominant in the early stages of recovery, but by 12 months people felt they were much more aware of the ups and downs of recovery for them, and they engaged in more nuanced strategies that allowed for the course of recovery within the context of their lives.

In contrast, the coding visualisation for the category "tension between life on hold and keeping up with life", showed that the six constituent codes all retained very stable prominence across the three timepoints. In this case, viewing the interview extracts revealed that participants discussed how the same concept within their understanding of their recovery subtly shifted in how it manifested over time. For example, several participants discussed the feeling that life had been put "on hold" as an important experience across all three time-points, but this went from a feeling of life activities being suddenly halted at 6 months post-injury, to a place where they were seeing life carrying on around them without 
their participation, and then to a realisation that in order to carry on, different choices and different pathways needed to be chosen - until then, the sense of being stalled remained.

\section{Enabling exploration of contextual differences in experiences}

Another facet of data visualisation was exploration of patterns in coding according to demographic characteristics of participants: specifically ethnicity, gender, age and injury severity. For example, interview extracts that discussed the difficulties associated with reduced income, reliance on a benefit or allowance, the burden of extra costs associated with recovery, and the problems associated with having to make financial choices between meeting basic needs versus doing things that make life meaningful were allocated to a code named "financial pressure". Using data visualisation, we could see the pattern in the prominence of this code for different ethnicities was different to the overall pattern in prominence when all ethnicities were combined. In particular, interviewees who identified as Māori (indigenous peoples of Aotearoa New Zealand) appeared to have a particularly large number of extracts in the code "financial pressure", while people who identified as New Zealand European had relatively fewer. When we explored this in the interview data, we identified that the aspects of financial pressures discussed by Māori and NZ European participants were quite different. Some Māori participants described extremely challenging financial situations - for example being "down to our last dollar" or "living in the car", while participants identifying as NZ European tended to talk about issues like not being able to meet the goals they had been working towards prior to their injury, or the difficulties faced in having to pay for non-funded treatments. This revealed important questions about how existing social and health disparities may intensify in the process of recovery and adaptation from TBI, and prompted us to explore the ways in which this was visible in our data. 
One of the challenges we experienced was setting up the data visualisation software to ensure it presented the data in a way that facilitated qualitative analysis. Data visualisation is often used to see numerical and statistical trends in large datasets, and the consultant we engaged to help develop the visualisation for our study was not accustomed to requests for "vague" visualisations of relative prominence. However, the software was able to be set up in a way that was very useful for our study by using colour density maps in the place of numbers (see the picture of our dashboard in supplementary file). There may well be other forms of maps and other visualisations that could be set up for different study topics for use by experienced qualitative researchers. However, as analysts, it was important that we viewed our maps as indicators of where to explore the detail in the data, not as findings in themselves. It is important to continue to be mindful of the potential dangers in working with data visualisation software. If the data visualisation dashboards were developed in a way that encouraged the researchers to see coding in terms of counts and ranking, and not enough attention was paid to the process of going back to the interviews, it could lead to inappropriate conclusions.

\section{Utilising sub-analyses}

In some instances, there were topics that needed to be subsumed within a broader category to stay consistent with the research question and analysis approach for the overall analysis, but the data showed potential for important insights in that area alone. In these cases, we utilised sub-analyses to ensure these insights were not lost due to the scope and size of the study. These were treated as separate analyses that followed up particular aspects of recovery and adaptation that emerged as important during the overall analysis. Some of these utilised the same methods of analysis but looked specifically at a particular topic in-depth (e.g. sleep issues (reference removed for peer review), experiences of health services), while others used the data collected for the study but employed a 
different methodology. Providing for sub-analyses that make use of a different methodology allowed exploration of a wider view of some of the difficulties encountered during the recovery and adaptation process. For example, a post-structural analysis focusing on 'discourses of recovery' examined the study data alongside media, clinical guidelines and academic texts in order to investigate the contribution of cultural and societal practices and norms in participants' experiences of accomplishments and challenges in their recovery. Wherever possible, members of the study team who had expertise in the specific topic or analysis methodology required undertook the sub study. These sub-analyses were regarded as separate studies and separate funding was sought where required.

\section{Conclusions}

This article extends existing literature on optimising data analysis for longitudinal qualitative research, particularly for research focused on health-related events. We offer a detailed description of analysis strategies used for a large longitudinal qualitative study focusing on a population with a complex trajectory, in which the inquiry was specifically targeted to processes (recovery and adaptation) that inherently involve re-interpretation and re-evaluation of experiences over time. These factors produced a distinctive range of considerations in facilitating appropriate data analysis, requiring a collection of techniques and technologies designed to help manage the process and exploit the volume and complexity of the data. The use of data management and data visualisation technologies for this study also provides an example of a move away from the routine use of specialised qualitative data management systems. Explicit consideration of a wider range of technologies, including those not specifically designed for qualitative research, opened up opportunities to handle data and view coding patterns in different ways. Furthermore, combining these technologies with manual concept mapping techniques and sub-analyses enabled a dynamic process by which we could review the details of coding 
and thematic groupings in the context of the emerging interpretation of the whole dataset, while maintaining an appreciation of the complexity that characterises recovery and adaptation after TBI. 


\section{References}

Becker, G. (1993). Continuity after a stroke: Implications of life-course disruption in older age. The Gerontologist, 33(2), 148-158.

Bidart, C. (2013). What does time imply? The contribution of longitudinal methods to the analysis of the life course. Time \& Society, 22(2), 254-273. doi:10.1177/0961463×12447493

Calman, L., Brunton, L., \& Molassiotis, A. (2013). Developing longitudinal qualitative designs: lessons learned and recommendations for health services research. BMC Medical Research Methodology, 13(14). doi:10.1186/1471-2288-13-14

Campbell, N., Murray, E., Darbyshire, J., Emery, J., Farmer, A., Griffiths, F., . . Kinmouth, A. (2007). Designing and evaluating complex interventions to improve health care. British Medical Journal, 334, 455-459. doi:10.1136/bmj.39108.379965.BE

Carlsson, E., Paterson, B. L., Scott-Findlay, S., Ehnfors, M., \& Ehrenberg, A. (2007). Methodological issues in interviews involving people with communication impairments after aquired brain damage. Qualitative Health Research, 17, 1361-1371. doi:10.1177/1049732307306926

Charmaz, K. (1995). The body, identity and self: Adapting to impairment. The Sociological Quarterly, 36(4), 657-680.

Charmaz, K. (2006). Constructing grounded theory : a practical guide through qualitative analysis. Los Angeles: SAGE.

Corden, A., \& Millar, J. (2007). Time and Change: A Review of the Qualitative Longitudinal Research Literature for Social Policy. Social Policy and Society, 6(04), 583-592. doi:doi:10.1017/S1474746407003910

Faircloth, C. A., Boylstein, C., Rittman, M., Young, M. E., \& Gubrium, J. (2004). Sudden illness and biographical flow in narratives of stroke recovery. Sociology of Health \& IIIness, 26(2), 242-261.

Faircloth, C. A., Rittman, M., Boylstein, C., Young, M. E., \& Van Puymbroeck, M. (2004). Energizing the ordinary: Biographical work and the future in stroke recovery narratives [Article]. Journal of Aging Studies, 18(4), 399-413. doi:10.1016/j.jaging.2004.06.006

Levack, W. M. M., Kayes, N. M., \& Fadyl, J. K. (2010). Experience of recovery and outcome following traumatic brain injury: a metasynthesis of qualitative research. Disability \& Rehabilitation, 32(12), 986-999.

Lloyd, V., Gatherer, A., \& Kalsy, S. (2006). Conducting qualitative interview research with people with expressive language difficulties. Qualitative Health Research, 16, 1386 - 1404. doi:10.1177/1049732306293846

Lowe, M., \& Molassiotis, A. (2011). A longitudinal qualitative analysis of the factors that influence patient distress within the lung cancer population. Lung Cancer, 74, 344-348. doi:10.1016/j.lungcan.2011.03.011.

Patton, M. Q. (2002). Qualitative research and evaluation methods (3rd ed.). California: Sage publications.

Pinnock, H., Kendall, M., Murray, S., Worth, A., Levack, P., Porter, M., . . Sheikh, A. (2011). Living and dying with severe chronic obstructure pulmonary disease: multi-perspective longitudinal qualitative study. BMJ, 342(d142). doi:10.1136/bmj.d142 
Qlik. (2015). Qlik's associative model: Seeing the whole story that lives within your data: White paper. Radnor, PA: QlikTech International AB.

Rubin, H. J., \& Rubin, I. S. (2005). Qualitative interviewing: The art of hearing data (2nd ed.). California: Sage.

Thomson, R. (2007). The Qualitative Longitudinal Case History: Practical, Methodological and Ethical Reflections. Social Policy and Society, 6(04), 571-582. doi:doi:10.1017/S1474746407003909

Thomson, R., \& Holland, J. (2003). Hindsight, foresight and insight: The challenges of longitudinal qualitative research. International Journal of Social Research Methodology, 6(3), 233-244. doi:10.1080/1364557032000091833

White, J. H., Gray, K. R., Magin, P., Attia, J., Sturm, J., Carter, G., \& Pollack, M. (2012). Exploring the experience of post-stroke fatigue in community dwelling stroke survivors: a prospective qualitative study. Disability \& Rehabilitation, 34(16), 1376-1384.

doi:10.3109/09638288.2011.645111 